\title{
Targeting asthma care in general practice using a morbidity index
}

\author{
K P Jones, I H Charlton, M Middleton, W J Preece, A P Hill
}

\begin{abstract}
Objectives-To evaluate a morbidity index as a postal surveillance tool in defining previously diagnosed asthmatic patients needing extra education or management; to determine the accuracy of a computerised asthma register in general practice.

Design-Postal questionnaire survey of asthmatic patients identified from a computer register. Questionnaire comprised three morbidity questions, two questions about current asthma status, and one about treatments.
\end{abstract}

Setting-Urban general practice of 8400 patients linked to academic unit.

Subjects -853 asthmatic patients of all ages.

Main outcome measures-Numbers of patients with low, medium, and high morbidity; associations of these groups with age, asthma status, and drugs taken.

Results - Two mailings yielded 621 replies (73\%); 28 patients $(5 \%)$ had moved away, leaving 593 for analysis. Attempts were subsequently made to contact $20 \%$ sample of non-respondents. 234 respondents $(40 \%)$ were in the "low morbidity" group, $149(25 \%)$ in the "medium morbidity" group, and $210(35 \%)$ in the "high morbidity" category. 53\% of patients perceiving themselves as currently asthmatic (193/362) were in the high morbidity group, but $7 \%(11 / 153)$ who said they were no longer asthmatic and $8 \%(6 / 78)$ who did not believe they had ever been asthmatic were also in that group. High morbidity was also found in $10 \%(18 / 185)$ of those on no treatment, $38 \%(59 / 154)$ of those on bronchodilators alone, and $54 \%(119 / 220)$ of those on inhaled corticosteroids. 25 patients $(4 \%)$ were wrongly identified as asthmatic; when combined with returns marked "gone away" this gave a disease register accuracy of $91 \%$.

Conclusions - This exercise identified subgroups of previously diagnosed asthmatic patients with high morbidity in general practice who might benefit from extra education and management and revealed some misclassification on the asthma disease register.

\section{Introduction}

Asthma is one of the most important chronic health

Primary Medical Care Group, Faculty of Medicine, University of Southampton, Aldermoor Health Centre,

Southampton SO1 6ST $\mathrm{K}$ P Jones, senior lecturer I H Charlton, research fellow M Middleton, research nurse W J Preece, practice nurse A P Hill, general practitioner

Correspondence to: $\mathrm{Dr}$ Jones.

BM于 1992;304:1353-6 asthma care in the community. Clearly it is within the realms of primary care that improvements in the management of asthma and the quality of life of its sufferers need to be made. ${ }^{14-16}$

The recent publication of guidelines for the management of asthma in both adults ${ }^{17} 18$ and children ${ }^{1920}$ together with the greater availability of peak flow meters on prescription have provided opportunity for innovations in primary care, such as nurse run asthma clinics $^{21}$ and patient self management plans. ${ }^{22}{ }^{23}$ Such new methods of caring for patients with asthma should prove beneficial for at least a proportion of asthma sufferers, but with roughly $10 \%$ of patients on all general practice lists likely to have this disease proactive care of this sort is not feasible for every patient. Methods of targeting extra care for asthma towards those most in need might avoid the problems experienced with cervical cytology screening - that is, those who regularly attend are those least at risk.

We have previously described a morbidity index derived from general practice data that has been shown to correlate with lung function. ${ }^{24}{ }^{25}$ This index seems to be suitable for opportunistic use and also for practice surveys using the computerised asthma disease registers which are now becoming common in general practice (35\% of Hampshire practices had an asthma register in 1991 (unpublished data)). The use of computers in general practice remains controversial ${ }^{26}$ and not without difficulties. ${ }^{27} 28$ The accuracy of these registers and the potential problems for patients when inaccuracies occur are thus worth exploring further.

This study investigates the usefulness of the morbidity index as a postal surveillance tool in defining those previously diagnosed asthmatic subjects who need extra education and management. It also examines the accuracy of a computerised disease register and the effects of the inaccuracies found.

\section{Population and methods}

The Aldermoor Health Centre urban practice of about 8400 patients has had an asthma register for more than 10 years. Patients are added to the register by the computer operator when the word "asthma" appears in the problem column of the A4 records, but are deleted only when they leave the list. The practice has had one or two doctors with a special interest in asthma since the early 1980 s and now offers a comprehensive nurse run asthma care service.

A simple one page questionnaire (see figure) was constructed on the Abies-Meditel practice computer. This could then be mail merged with the names and addresses of all those patients recorded as asthmatic on the computer so that individualised letters could be produced. The first mailing went out in March 1991 with a follow up to non-respondents about four weeks later. The manual case notes of a sample of patients selected by using random number tables from the high morbidity group were examined to ascertain their recent consulting patterns. 


\section{Aldermoor Health Centre \\ Aldermoor Close \\ Southampton SO1 6ST \\ Tel: 0703-783111}

Dear Patient

Recently we have had the opportunity to update our computer at the Health Centre and our records indicate that you have asthma or a tendency to asthma now or some time in the past. Firstly, we would like to check that our records are correct and then to ask how well you feel that your asthma has been controlled recently.

We would be grateful if you could answer the following:

1. Do you think you have asthma now? Yes/No

2. Have you ever been told you have asthma by a doctor or nurse some time during your life?

Yes/No

Regardless of the previous responses could you answer the following:

3. Are you in a wheezy or asthmatic condition at least once per week?

Yes/No

4. Have you had time off work or school in the last year because of wheeze or asthma?

Yes/No/Not applicable

5. Do you suffer from attacks of wheezing during the night?

Yes/No

6. Would you please list any medications that you are currently prescribed.

If there is anything in the questionnaire that you would like to discuss, please feel free to contact the surgery and speak to Sister Preece or Sister Middleton.

Thank you for your help.

Survey questionnaire

The case records of all those in the "asthma never" group were also examined to discover whether asthma was mentioned therein. Attempts were made subsequently to contact a $20 \%$ sample (also by using random number tables) of those still not replying (by telephone, further letter, or home visit). Some patients did not answer the question on treatment. Their case notes were examined to elucidate which treatment group they should be placed in.

Encoded data including the patient's age in completed years were entered on an IBM compatible personal computer and analysed by using SPSS PC+ version 3.0.

\section{Results}

Six hundred and twenty one replies were received from the two mailings, giving a response rate of $73 \%$. Twenty eight patients $(5 \%)$ had moved away, leaving 593 for analysis (table I). The relations between the morbidity index and patients' perceptions of their asthma status (asthma now, asthma in the past, asthma never), antiasthma drugs taken, and age are shown in tables II, III, and IV respectively. An analysis of the morbidity index in inhaled steroid takers did not show any significant trend with age $\left(\chi^{2}=1 \cdot 25 ; p=0 \cdot 97\right)$.

Of the 78 patients in the asthma never group, one had bronchiectasis, 15 were definitely asthmatic at some stage, 25 were keystroke errors on the computer -that is, there was clearly no mention of asthma anywhere-seven had chronic obstructive airways disease or emphysema, four had hay fever or chronic rhinitis, and the remaining 26 had a variety of probably asthmatic labels, such as "? asthma," "verv mild asthma," "hay asthma," "asthma with hay fever," "horse asthma," and "asthma with allergies."
Analysis of the case records of the 18 patients selected from those in the high morbidity group showed that only three were currently under the care of one of the doctors in the practice for attempts to improve asthma control. Fourteen had been seen for some other reason than asthma in the previous six months, seven patients had been seen in the previous three months for asthma, and eight more in the previous year. Four patients had not been seen at all in the previous year.

Fifty patients were selected by means of random number tables from those who had not responded to the two mailed questionnaires and further attempts made to contact them. Twenty seven further completed replies were obtained with seven more patients gone away, one questionnaire mislaid, and the remaining 15 patients not contactable despite remaining on our list and several attempts at reaching them (including home visits). Seventeen of these slow responders were in the "asthma now" group, seven in the "asthma in past" group, and three in the "asthma never" group. Seven were in the low morbidity category, 10 in the medium

TABLE I - Results of postal questionnaire survey (593 patients available for analysis)

\begin{tabular}{lc}
\hline & No (\%) of patients \\
\hline Admitted to having asthma now & $362(61)$ \\
Past but not current asthma & $153(26)$ \\
No self recognised history of asthma & $78(13)$ \\
Wheezy or asthmatic at least once a week & $246(41)$ \\
Time off work or school in past year through & $125(21)$ \\
wheeze or asthma & $116(20)$ \\
Not attending work or school & $249(42)$ \\
Suffer from attacks of wheezing during night & $234(40)$ \\
Low morbidity (as defined by our index x $\left.^{24}\right)$ & $149(25)$ \\
Medium morbidity & $210(35)$ \\
High morbidity & $185(31)$ \\
Taking no antiasthma treatment at all & $154(26)$ \\
Bronchodilators only & $220(37)$ \\
Inhaled steroids and bronchodilators & $22(4)$ \\
Non-steroidal prophylactic drugs & $12(2)$ \\
Steroid and non-steroidal agents & \\
\hline
\end{tabular}

TABLE II - Morbidity index by asthma status as perceived by patients (figures are numbers (percentages) of patients)

\begin{tabular}{lrccc}
\hline & \multicolumn{4}{c}{ Morbidity index } \\
\cline { 2 - 5 } Self perceived asthma status & Low & Medium & High & Total \\
\hline Asthma now & $57(16)$ & $112(31)$ & $193(53)$ & $362(100)$ \\
Asthma in past & $110(72)$ & $32(21)$ & $11(7)$ & $153(100)$ \\
No asthma ever & $67(86)$ & $5(6)$ & $6(8)$ & $78(100)$ \\
\hline
\end{tabular}

TABLE III - Morbidity index by drugs used (numbers (percentages) of patients)

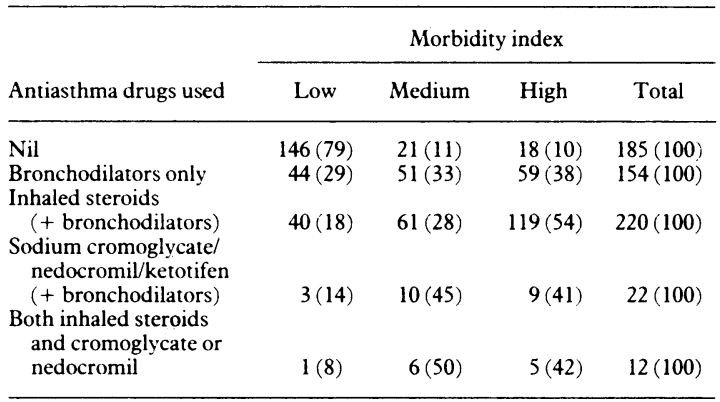

TABLE IV-Morbidity index byage (numbers(percentages) of patients)

\begin{tabular}{lrrrr}
\hline & \multicolumn{4}{c}{ Morbidity index } \\
\cline { 2 - 5 } Age in completed years & Low & Medium & High & \multicolumn{1}{c}{ Total } \\
\hline $0-4$ & $10(38)$ & $8(35)$ & $7(27)$ & $25(100)$ \\
$5-15$ & $75(45)$ & $35(21)$ & $57(34)$ & $165(100)$ \\
$16-40$ & $92(41)$ & $60(27)$ & $71(32)$ & $223(100)$ \\
$41-65$ & $37(33)$ & $28(25)$ & $47(42)$ & $112(100)$ \\
$>65$ & $19(30)$ & $18(28)$ & $27(42)$ & $64(100)$ \\
\hline
\end{tabular}

^Age missing for two patients. 
morbidity category, and 10 in the high morbidity category. Ten patients were on no medication, two on bronchodilators alone, 14 on inhaled steroids, and one on cromoglycate.

\section{Discussion}

The use of the morbidity index principle in this postal survey of asthmatic patients identified a group with self reported high morbidity. Our previous work illustrating the relation of the index to spirometric measures and diurnal variation in peak expiratory flow rate suggested that this group had, on average, worse asthma than people in the other groups. ${ }^{24} 25$ These patients are therefore among those most in need of extra help with management-although the index is bound to miss some asthmatic patients with poorly controlled disease due to completion bias and imperfect sensitivity. The group may also include some patients with other forms of lung disease.

\section{PATIENTS' PERCEPTIONS OF ASTHMA}

The study also gives interesting information concerning the associations of self reported high morbidity. Understandably, most patients $(53 \%$; 193/362) who believed they had current asthma ("asthma now" group) were those with continuing symptoms and thus were found in the high morbidity group. Rather more surprisingly, $7 \%(11 / 153)$ of those in the "asthma in past" group still made the high morbidity group as did $8 \%(6 / 78)$ of those who believed they had never had the disease. This might have been due to deficiencies in our questionnaire, but poor perception of asthma or its denial by the patient or poor communication from health care workers may also be important.

The great majority of patients on no treatment $(79 \%$; 146/185) reported low morbidity, but there was still a small group $(10 \% ; 18 / 185)$ who placed themselves in the high morbidity group. The position was worse in the bronchodilators alone group, with $38 \%$ of patients $(59 / 154)$ with high morbidity, and worst of all in the steroid group, where the majority of patients $(54 \%$; 119/220) had high morbidity despite treatment.

A number of conclusions can be drawn from these observations, though they are in part dependent on an assumption of the keeping of good notes and registers. Patients clearly have different perceptions of "having asthma" than their health carers, since they clearly equate having symptoms with having the disease and conversely feel that the disease is not present when they are asymptomatic. As we now know that chronic inflammatory changes exist even in asymptomatic asthmatic patients, ${ }^{29}$ this is clearly one issue where our patients' understanding urgently needs to be improved. Some asthma sufferers seem also to be accepting a continuing burden of morbidity as normal for them and may thus be "turning down their life thermostat" to accept a poorer quality of life than might be achieved. Many patients using bronchodilators might benefit from inhaled steroids if the British Thoracic Society guidelines ${ }^{17}{ }^{18}$ are followed.

\section{RELATION BETWEEN MORBIDITY AND STEROIDS}

The persistence of high morbidity despite use of inhaled steroids in $20 \%$ of patients overall was worrying. Patients may not be complying with their medicines ${ }^{30}$ or their inhaler technique may be poor, as shown in a number of other studies. ${ }^{31-34}$ Their prescribed dose of steroid may be too low. It may be that this high percentage of high morbidity patients merely reflects that steroids tend to be given to those with more symptoms and thus more severe asthma. Current steroid therapy may not be sufficient to control disease in all asthmatic patients. The argument that a sizable number of these high morbidity steroid taking patients have fixed airways obstruction and thus continuing symptoms is not borne out by the age analysis of this group. There was no preponderance of high morbidity steroid takers in the older age range, which might be expected if this group was in reality dominated by patients with chronic obstructive airways disease, although this possibility cannot be completely excluded.

The data obtained from the non-respondents clearly indicated that this group largely comprised asthmatic patients with medium or high morbidity and receiving inhaled steroids. Thus it is a reasonable assumption that the whole group of non-respondents was certainly not dominated by low morbidity and that the fully analysed data and the conclusions reached reflected an acceptable sample of the whole study population.

\section{ACCURACY OF REGISTER}

We also set out to test the accuracy of our computerised asthma register. When the number of replies marked "gone away" (28) was added to those definitely identified as included due to computer keystroke error (25) this gave an accuracy of $91 \%$ when applied to our sample. The exclusion of patients with chronic obstructive airways disease, emphysema, hay fever, and chronic rhinitis would reduce this figure only to $90 \%$.

This sort of error factor needs to be borne in mind when making interpractice comparisons of asthma prevalence based on the size of asthma registers. There are clearly other issues at stake. Firstly, outreach of the kind described here is a fairly costly exercise, and such costs are inevitably raised if letters are sent to "ghosts" on the list. We estimate that about 40 hours of researchers' time and $£ 500$ in stationery and postage were required for the whole project.

A second and more important issue was the disturbance to patients if they are wrongly identified as being asthmatic. We received a number of telephone calls and letters from irate patients who were unhappy to find we thought they had asthma. Others may have been similarly disquieted without making contact. Raising anxieties in a minority of patients may be acceptable if clear benefits follow for the majority, but evidence of such benefit is yet to emerge.

\section{UNMET NEED}

The patients reporting high morbidity represented $2.5 \%$ of the practice list $(210 / 8400)$. This is still too high a number for individual proactive care from doctors or nurses to be easily provided in the context of busy general practice. An initial assessment of such patients would require roughly 140 hours and a three monthly review - a further 140 hours a year based on an average of 40 minutes per assessment and 10 minutes per review. However, it is clear from our small audit of medical supervision of a sample of such patients that there is a need which requires addressing. Opportunistic surveillance, though possible as seen from the consultation data on the small number of high morbidity case records sampled, does not seem to be occurring. Accordingly, we have set up a series of evening educational group meetings for a sample of the high morbidity patients which we feel can be successfully resourced from within the practice at least in the short term. We shall be assessing the impact, if any, of this approach on morbidity and consulting patterns in due course. For asthma surveillance of this sort to be justified it is essential that the process can be shown to lead to reductions in morbidity. If tangible benefits do not emerge we may have raised expectations but provided little real improvement.

In conclusion, therefore, the use of the morbidity index as a tool for asthma surveillance in general practice has highlighted a group of previously diag- 
nosed asthmatic patients who might benefit from extra education and management. This group would not have been so identified if other criteria such as current perception of asthma or class of drug taken had been used instead, nor if a principle of continuing to use opportunistic review had been adopted. The index thus seems worthy of more widespread use in primary care. Further validation of this approach is in progress by interviewing samples of high and low morbidity patients to determine what factors may be associated with variations in morbidity. Some of these may be obvious, such as treatment compliance, inhaler technique, and smoking status, but others may yet surprise us.

KPJ was a Sir Jules Thorn research fellow during this study. We thank Michelle Pettet for help with the practice computer, Elaine Jones for help with encoding the data, and Dr Ann-Louise Kinmonth for useful comments on the manuscript. This paper was presented as a poster at the winter 1991 meeting of the British Thoracic Society.

1 Burney PGJ. Asthma mortality in England and Wales: evidence for a further increase. Lancet 1986;ii:323-6.

2 Evans R, Mullally DI, Wilson RW, Gergen PJ, Rosenberg HM, Grauman JF, et al. National trends in the morbidity and mortality of asthma in the US. Prevalence, hospitalization and death from asthma over two decades: 1965-1984. Chest 1987:91:65-74S.

3 Mao Y, Semenciw R, Morrison H, MacWilliams L, Davies J, Wigle D. Increased rates of illness and death from asthma in Canada Can Med Assoc $\mathcal{F}$ 1987;137:620-4.

4 Sly RM. Mortality from asthma. 7 Allergy Clin Immunol 1988;82:705-17

5 Anderson HR. Trends and district variations in the hospital care of childhood asthma: results of a regional study 1970-85. Thorax 1990;45:431-7.

6 Mitchell EA. International trends in hospital admission rates for asthma. Arch Dis Child 1985;60:376-8.

7 Burney PGJ, Papacosta AO, Withey CH, Colley JRT, Holland WW. Hospital admission rates and the prevalence of asthma symptoms in 20 local authority districts. Thorax 1991; $46: 574-9$.

8 Burney PGJ, Chinn S, Rona RJ. Has the prevalence of asthma increased in children? Evidence from the national study of health and growth 1973-86. BMF 1990;300:1306-10

9 Hilton S, Anderson HR, Sibbald B, Freeling P. Controlled evaluation of the effects of patient education on asthma morbidity in general practice. Lancet $1986 ; 1: 26-9$.

10 White PT, Pharoah CA, Anderson HR, Freeling P. Randomized controlled trial of small group education on the outcome of chronic asthma in general practice. 7 R Coll Gen Pract 1989;39:182-6.

11 Turner-Warwick $M$. Nocturnal asthma: a study in general practice. $\mathcal{F} R \mathrm{Coll}$ Gen Pract 1989;39:239-43.

12 Horn CR, Cochrane GM. An audit of morbidity associated with chronic asthma in general practice. Respir Med 1989;83:71-5.

13 Charlton IH, Bain DJG. The care of children with asthma in general practice: signs of progress?. Br f Gen Pract 1991;41:256.

14 Jones KP. Asthma-still a challenge for general practice. $f R$ Coll Gen Praci 1989;39:254-6.

15 Anonymous. Management of asthma in the community. Lancet 1989;ii: 199-200.

16 Jones KP. Asthma care in general practice-time for revolution?. Br $\mathcal{G}$ Ge Pract 1991;41:224-6.

17 British Thoracic Society. Guidelines for management of asthma in adults: I chronic persistent asthma. BMF 1990;301:651-3.

18 British Thoracic Society. Guidelines for management of asthma in adults: II acute severe asthma. BMF 1990;301:797-800.

19 Warner JO, Gotz M, Landau LI, Levison H, Milner AD, Pedersen S, Silverman M. Management of asthma: a consensus statement. Arch D is Child 1989;64: 1065-79.

20 Asthma: a follow up statement from an international paediatric consensus group. Arch Dis Child 1992;67:240-8.

21 Charlton IH, Charlton G, Broomfield J, Mullee MA. Audit of the effect of a nurse run asthma clinic on workload and patient morbidity in a general practice. Br $\mathcal{J}$ Gen Pract 1991;41:227-31.

22 Beasley R, Cushley $M$, Holgate ST. A self management plan in the treatment of adult asthma. Thorax 1989;44:200-4.

23 Charlton I, Charlton G, Broomfield J, Mullee M. Evaluation of peak flow and symptoms only self-management plans for control of asthma in genera practice. $B M \mathcal{F} 1990 ; 301: 1355-9$.

24 Jones KP. Correlates of asthma morbidity in general practice. Thorax 1991;46:318-9P.

25 Jones KP, Bain DJG, Middleton M, Mullee MA. Correlates of asthma morbidity in primary care. $B M 7$ 1992;304:361-4.

26 Stanley PH. We don't have a computer. BMf 1991;303:971-2.

27 Johnson N, Mant D, Jones L, Randall T. Use of computerised general practice data for population surveillance: comparative study of influenza data. $B M \mathcal{Z}$ 1991;302:763-5.

28 Jick H, Jick SS, Derby LE. Validation of information recorded on general practitioner based computerised data resource in the United Kingdom. BMf 1991;302:766-8.

29 Beasley R, Roche WR, Roberts JA, Holgate ST. Cellular events in the bronch in mild asthma and after provocation. Am Rev Respir Dis 1989;139:806-17.

30 Horn CR, Clark TJH, Cochrane GM. Compliance with inhaled therapy and morbidity from asthma. Respir Med 1990;84:67-70.

31 Crompton GK. Problems patients have using pressurized aerosol inhalers. Eur f Respir Dis 1982;63:101-4.

32 Horsley MG, Bailie GR. Risk factors for inadequate use of pressurized aeroso inhalers. F Clin Pharm Ther 1988;13:139-43.

33 Allen S, Prior A. What determines whether an elderly patient can use metered dose inhaler correctly: $B r f$ Dis Chest 1986;80:45-9.

34 Hilton S. An audit of inhaler technique among asthma patients of 34 general practitioners. Br f Gen Pract 1990;40:505-6.

(Accepted 7 April 1992)

\section{MATERIA NON MEDICA}

\section{Tabanka in Tobago-some verbal accounts}

Tabanka is a term used in Trinidad and Tobago for a characteristic disturbed state of mind, owing to the loss of a sexual partner, typically affecting males with social aspirations of upward mobility. Sufferers exhibit exaggerated grief, neglect themselves, behave bizarrely in public, and preoccupy themselves with their lost partner. Tabanka invariably causes great hilarity among bystanders.

I asked several Tobagonians about tabanka:

Food stall minder, male, about 40- "It's due to the clash between the old slave culture and the Western way of life. One guy you see round here, intelligent fellow, going with a schoolteacher, she left him, and now he just hangs around the airport with his guitar looking for her. Men like me who don't get involved, we're safe. I'm a wanderer, come and go as I please."

Professor of A frican studies working in USA, male, aged 50 or over - "It's a situation where a woman has a man under a spell - he has no self control because of her, his life is not his own. It's a form of love sickness." (This was the only explanation mentioning a magical cause.)

Rum shop owner, male, aged 25 - "Ha, tabanka. It affects some folks, they turn to rum or go mad, never recover. Doesn't affect women. It's because we're getting married age 18, 20 here in Tobago. Fellow has a child with a girl, likes to show off, then she leaves him and he gets to studying on it, never recovers. Now me, I understand women, that will never happen to me."

Community psychiatric nurse, aged 40, female-"They don't come into the psychiatric clinic for treatment."

Maid, aged 22-(Laughs) "Tabanka-who told you about that? Policemen can get that."

Medical officer, aged 35, female-(Laughs) "Tabanka. It's not like an illness, they don't get to need treatment."

Tabanka seems to be a culturally sanctioned form of outlet. It is readily recognised locally and the sufferer's personal circumstances are public knowledge, perhaps protecting the victim from being detained by the authorities as local standards of public behaviour are strict.

The island's social hierarchy has many subtle layers. In the carnival tradition of ribald satirical calypso aimed at deflating pompous public figures, hilarity is caused by the spectacle of a failed, vain attempt to climb the social ladder into middle class respectability through a relationship, and figures of authority such as policemen and teachers are particularly vulnerable.

Most other "culture concentrated" disorders are rage, fear, or dissociation states: tabanka in contrast may be a bereavement reaction that has not been "medicalised" by local culture.-STEFAN CEMBROWICZ, general practitioner, Bristol 